\section{Effect of Psychoactive Drugs on Demographic Parameters of the Blow Fly Chrysomya albiceps (Wiedemann) (Diptera: Calliphoridae)}

Juliana Zibordi Gião', Carolina Reigada ${ }^{2}$, Thiago de Carvalho Moretti ${ }^{3 *}$ and Wesley Augusto Conde Godoy ${ }^{2}$

${ }^{1}$ Department of Parasitology, Institute of Biosciences, São Paulo State University Julio de Mesquita Filho (UNESP), Botucatu, SP, Brazil

${ }^{2}$ Department of Entomology and Acarology, Luiz de Queiroz College of Agriculture, University of São Paulo (USP), Piracicaba, SP, Brazil ${ }^{3}$ Department of Animal Biology, Institute of Biology, State University of Campinas (UNICAMP), Campinas, SP, Brazil

\begin{abstract}
Knowledge of the factors influencing the ecology of blow flies, especially the dynamic population equilibrium, is essential in forensic entomology. However, lack of knowledge of the action of psychoactive drugs on the population dynamics of these flies may affect the inferences that experts must make in medico-legal reports. This study evaluated the effects of amphetamine and phenobarbital on the population dynamics of Chrysomya albiceps (Wiedemann), by combining laboratory experimentation and mathematical modelling. Only amphetamine and its control, methanol, influenced the qualitative dynamic behaviour of Chrysomya albiceps, stabilising chaotic populations. The results are discussed in an ecological and forensic context.
\end{abstract}

Keywords: Carrion; Entomotoxicology; Flies; Forensic entomology; Forensic toxicology; Population dynamics

\section{Introduction}

The main goal of medico-criminal entomology is to provide evidence of the length of time that a body has been exposed to colonisation by arthropods, which helps to estimate the minimum post-mortem interval (PMI min) [1]. The PMI min is often determined by calculating the age of the oldest of the insect life stages collected from a corpse and/or crime scene, which are usually immature stages of blow flies (Diptera: Calliphoridae) [2]. However, errors in these estimates may occur if the larvae are desiccated, frozen [3], evaluated with inaccurate temperature models [4], or have absorbed chemical substances $[5,6]$. Other factors such as intra- or interspecific interactions and trophic interactions should also be considered, since they may also affect the development time, weight, size, and co-occurrence of related species [7-9].

The deaths related to drug use, or to accidental or deliberate use of poisons or toxic substances, explain the interest in the field of entomotoxicology. This branch of forensic science, which is especially useful when a corpse is in an advanced state of decomposition or skeletonised and its tissues and fluids are no longer suitable for toxicological analyses $[5,10]$, deals with the investigation of the effects of drugs and toxins present in human tissues on the biology of necrophagous insects. Seminal studies have indicated that the presence of toxic substances can affect the development of necrophagous insects, for instance altering the development time in larvae of blow flies $[11,12]$, muscid flies [13] and flesh flies [14-16].

According to the Brazilian Toxico-Pharmacological Information System (Sinitox), in 2014, medications headed the list of toxic agents that caused poisoning in humans in Brazil (30.37\%). Amphetamine acts mainly on the central nervous and cardiovascular systems [17]. This drug is used as an appetite moderator, but when mixed with other substances, it is transformed into methamphetamine-like stimulants, which are commonly used by commercial truckers to inhibit sleep, especially in Brazil. Fatal overdoses are rare [17]. Phenobarbital, a barbiturate, depresses the central nervous system and is used to treat seizure disorders [18]. Because of the narrow safety margin between therapeutic and toxic doses, barbiturates are often lethal in overdoses [19]. Alcohol or other agents that depress the central nervous system may, when combined with phenobarbital, enhance its action [18]. Barbiturates ranked in second place among the drugs used in suicide attempts in the state of Goiás, Central-West Brazil, in 2003-2004 [20].

Blow flies (Diptera: Calliphoridae) are among the first insects to perceive and colonise vertebrate remains [2,21]. Chrysomya albiceps (Wiedemann) originated in the Old World tropics, and over time spread around the world, reaching Brazil in 1975 or 1976 [22,23]. The species is of major forensic importance, allowing the estimation of the PMI min [21] and also the detection of ante-mortem use of drugs and toxins [24]. The establishment of Chrysomya species in Brazil affected the dynamic behaviour of native blow flies, causing the decline of populations of Cochliomyia macellaria (Fabricius) and, to a lesser extent, Lucilia eximia (Wiedemann) [25].

Calliphorid flies breed in discrete generations, displaying high sensitivity in demographic parameters influenced by larval density, which are usually closely associated with population equilibrium [25]. Mathematical models involving the analysis of fly population growth are powerful analytical tools for describing the long-term effects of ecological and behavioural patterns that are important for population dynamics, which are especially relevant for the demographic interpretation of species of medical-veterinary and forensic importance [25].

The theory of population dynamics of single species has been applied extensively over the last 20 years to study both introduced and native blow fly species, in order to better understand aspects of the population biology of each species $[25,26]$. These studies have revealed important differences in the dynamics of population equilibrium

*Corresponding author: Thiago de Carvalho Moretti, Department of Anima Biology, Institute of Biology, State University of Campinas (UNICAMP), Campinas, SP, Brazil, Tel: 551935216275; E-mail: tcmoretti@yahoo.com.br

Received November 13, 2017; Accepted November 21, 2017; Published November 25, 2017

Citation: Gião JZ, Reigada C, Moretti TC, Godoy WAC (2017) Effect of Psychoactive Drugs on Demographic Parameters of the Blow Fly Chrysomya albiceps (Wiedemann) (Diptera: Calliphoridae). J Forensic Res 8: 400. doi 10.4172/2157-7145.1000400

Copyright: ( 2017 Gião JZ, et al. This is an open-access article distributed under the terms of the Creative Commons Attribution License, which permits unrestricted use, distribution, and reproduction in any medium, provided the original author and source are credited. 
between introduced and native species [27]. Mathematical models that incorporate fecundity and survival as density-dependent processes and the spatial structure have been used to investigate the spatio-temporal dynamics of Chrysomya (introduced) and Cochliomyia (native) species [28]. The results indicate that Chrysomya species exhibit a stable 2-point limit cycle characterised by an oscillation between two values-a maximum and a minimum-which are representative of the population size as a function of time. Cochliomyia macellaria and Lucilia eximia exhibit a steady monotonic equilibrium, which stabilises the population size towards a single value [27].

The demographic parameters involved in these mathematical models are influenced by many factors, among which is the possible influence of psychoactive drugs. Modelling biological populations of blow flies is not a simple task [29], even when the action of drugs on biological and demographic parameters is not taken into account. Thus, the inclusion of this parameter in the system increases the complexity of the analysis, requiring the use of highly sensitive techniques in order to incorporate all the important processes into the system.

Little is known about the dynamic behavior of blow flies when they come into contact with food sources containing chemical substances that can in some way become incorporated into the insect's physiological system. This lack of information encompasses both laboratory experimentation and mathematical modelling applied to population dynamics. Studies performed to date have dealt exclusively with the influence of chemical substances on larval development time $[11,12,16]$ and, more rarely, on insect successional patterns [30].

Because environmental conditions (e.g., the presence of drugs, hormones or heavy metals) experienced in preceding generations can, theoretically, influence life-history characteristics of their offspring [31-33], the action of drugs may affect the dynamic behaviour of necrophagous flies, altering their patterns of population fluctuation as well as the dynamics of interspecific interactions, with possible implications for the composition of necrophagous fauna. Therefore, lack of knowledge of the effect of drugs on population dynamics of blow flies may potentially impair the quality of the inferences made by crime-scene professionals.

The objective of this study was to investigate the population dynamics of Chrysomya albiceps under the influence of two psychoactive drugs, amphetamine and phenobarbital.

\section{Material and Methods}

\section{Collection and maintenance of specimens}

Experimental populations of Chrysomya albiceps were obtained from specimens collected with hand nets on the campus of São Paulo State University, located at Botucatu, State of São Paulo, Brazil $\left(22^{\circ}\right.$ 53' 09" S; $048^{\circ} 26^{\prime} 42^{\prime \prime} \mathrm{W}$ ). Rodent carcasses were used as an attractive substrate during the collections. The collected specimens were anaesthetised at low temperature, identified to species, and placed in wood-framed cages with nylon mesh $(30 \times 30 \times 30 \mathrm{~cm})$ to form the laboratory populations. The experimental populations were fed sugar and water ad libitum and were maintained in $25 \pm 1^{\circ} \mathrm{C}$, a 12 hour photoperiod, and 70\% R.H. Adult females were fed beef liver as a supplemental protein source.

\section{Food substrate and insertion of drugs}

The food substrate used for the larval development of the blow flies was an artificial medium of milk powder, agar, brewer's yeast, Nipagin and casein [34], supplemented with chicken heart to enhance the development of the flies [35]. Treatments consisted of addition of psychoactive drugs to the artificial medium, contrasting with the regular artificial medium (control) as follows. A dose of $150 \mathrm{mg} / \mathrm{kg}$ of phenobarbital was used in the artificial diet as the first treatment. Amphetamine was the second treatment, taking into account the therapeutic dose of $0.07 \mathrm{mg} / \mathrm{kg}$. The amphetamine used in this study, which was the standard brand produced by Radian Corp., was purchased pre-diluted by the manufacturer, in methanol in a proportion of 1:1. To control for the amphetamine results, the experiments were also performed with methanol for subsequent comparison.

\section{Experimental design}

Exploratory intraspecific larval competition was established in the laboratory by setting six larval densities, 100, 200, 400, 600, 800 and 1000, with two replicates each, developed on a $50 \mathrm{~g}$ diet for the control, a $50 \mathrm{~g}$ diet with phenobarbital $(150 \mathrm{mg} / \mathrm{kg})$, a $50 \mathrm{~g}$ diet with amphetamine $(0.07 \mathrm{mg} / \mathrm{kg})$, and a $50 \mathrm{~g}$ diet with methanol $(0.07 \mathrm{mg} /$ $\mathrm{kg}$ ). This set of larval densities is suitable for simulating the process of larval competition in species of the genus Chrysomya, producing a decrease in demographic parameters as a function of density [28]. Larvae surviving to the adult stage produced both males and females. The fecundity was estimated by counting the number of eggs per female, expressed as the daily mean number of eggs, which is based on the length of the gonotrophic cycle of species of Chrysomya [36]. In order to estimate the fecundity, emerging adults received fresh bovine liver for a week to stimulate ovarian development, because they require additional protein to complete their gonotrophic cycle [36]. During this period, males and females also mated. A minimum of 20 females in each density were taken randomly from the cages. The insects were killed and dissected to record the number of eggs per female. Survival was estimated from the number of adults emerging from larvae reared at each density.

\section{Population dynamics}

Exponential regressions for fecundity and survival as a function of larval density were fitted to the data, since previous studies have confirmed their analytical suitability, producing a better fit than linear or hyperbolic regressions [28]. The decline in fecundity as a function of larval density can be visualised biologically as a Poisson process, which is usually described by the exponential function [37].

\section{Mathematical model}

The population dynamics was investigated by using bifurcation diagrams in the Prout and McChesney mathematical model [38] to describe the variation in population sizes due to the parametric space of fecundity and survival of Chrysomya albiceps.

The Prout and McChesney model has been extensively employed to describe the population dynamics of blowflies, emphasizing several demographic aspects and dispersal of native and exotic species [23,25,26,39]. The Prout and McChesney model [38] considers the effect of the delay due to larval competition in generation $t$ on the parameters of fecundity $(\mathrm{Fec})$ and survival (Surv) in the subsequent generation $(t+1)$, which is therefore a first-order finite-difference equation, written as:

$$
n_{t+1}=0.5 F e c\left(n_{t}\right) \operatorname{Surv}\left(n_{t}\right) n_{t}
$$

where 0.5 indicates that half of the population comprises females.

The variation in fecundity and survival rates as a function of the larval density was estimated by exponential regression, which can be 
written as $F e c=F^{*} e^{-f n}$ for fecundity and as $\operatorname{Surv}=S^{\star} e^{-s n}$ for survival. $F^{\star}$ and $S^{*}$ are the intercepts of the regression, which represent maximum (theoretical) values of fecundity and survival, and $f$ and $s$ are the coefficients of regression that estimate the rate of variation in fecundity and survival as a function of the density of immatures. Thus, by applying the exponential functions to equation [1] one has:

$$
n_{t+1}=0.5 \text { Fec }^{*} \operatorname{Surv}^{*} e^{-(f+s) n_{t}} n_{t} \quad[2]
$$

The general expression for the eigenvalue $[\lambda]$ associated with equation [1] is obtained by the derivative of $\mathrm{N}_{t+1}$ in relation to $\mathrm{N}_{t}$, evaluated at $k$, which describes the number of immatures in equilibrium. Thus, the eigenvalue $(\lambda)$ determines the type of population equilibrium, using equation [3]:

$$
N_{t+1}=1+\left.0.5 k S(k) \frac{d F\left(n_{t}\right)}{d N_{t}}\right|_{n_{t}=k}+1+\left.0.5 k F(k) \frac{d S\left(n_{t}\right)}{d N_{t}}\right|_{n_{t}=k}
$$

The dynamic behavior of Chrysomya albiceps can then be deduced from equation [3]. If $(\lambda)$ is less than 1 , in the module, then the equilibrium is stable and monotonic. Values more than 1 denote cyclic behaviours and even deterministic chaos.

\section{Results and Discussion}

The numerical simulations performed with the Prout and
McChesney [38] mathematical model, including all the treatments, revealed that changes in the qualitative dynamic behaviour of Chrysomya albiceps in response to different values of fecundity (Figure 1A-1D) and survival (Figure 2A-2D) were observed only with the addition of amphetamine and methanol to the rearing medium. Phenobarbital produced no qualitative change in the Chrysomya albiceps populations. A bifurcation diagram containing a sequence of dynamic behaviors, starting with a stable equilibrium, followed by limit cycles and unpredictable oscillations as a function of fecundity can be observed for the control (Figure 1A). The addition of methanol or amphetamine to the rearing medium stabilized the Chrysomya albiceps dynamic behavior (Figure 1C and 1D).

On the other hand, this effect was not observed for survival, suggesting no qualitative change in this parameter, since the four-point limit cycle observed in the control was the same as that found after the addition of methanol and amphetamine to the rearing medium (Figure $2 \mathrm{C}$ and $2 \mathrm{D})$.

The exponential regression analysis evaluating the variation of fecundity and survival in response to larval density performed in this study indicated that the intraspecific larval density exerts a significant negative effect on the demographic parameters obtained from the control and drug-treatment experiments (Table 1). The lowest fecundity intercept value was obtained for the control, and the highest value was observed in the amphetamine treatment (Table 1).
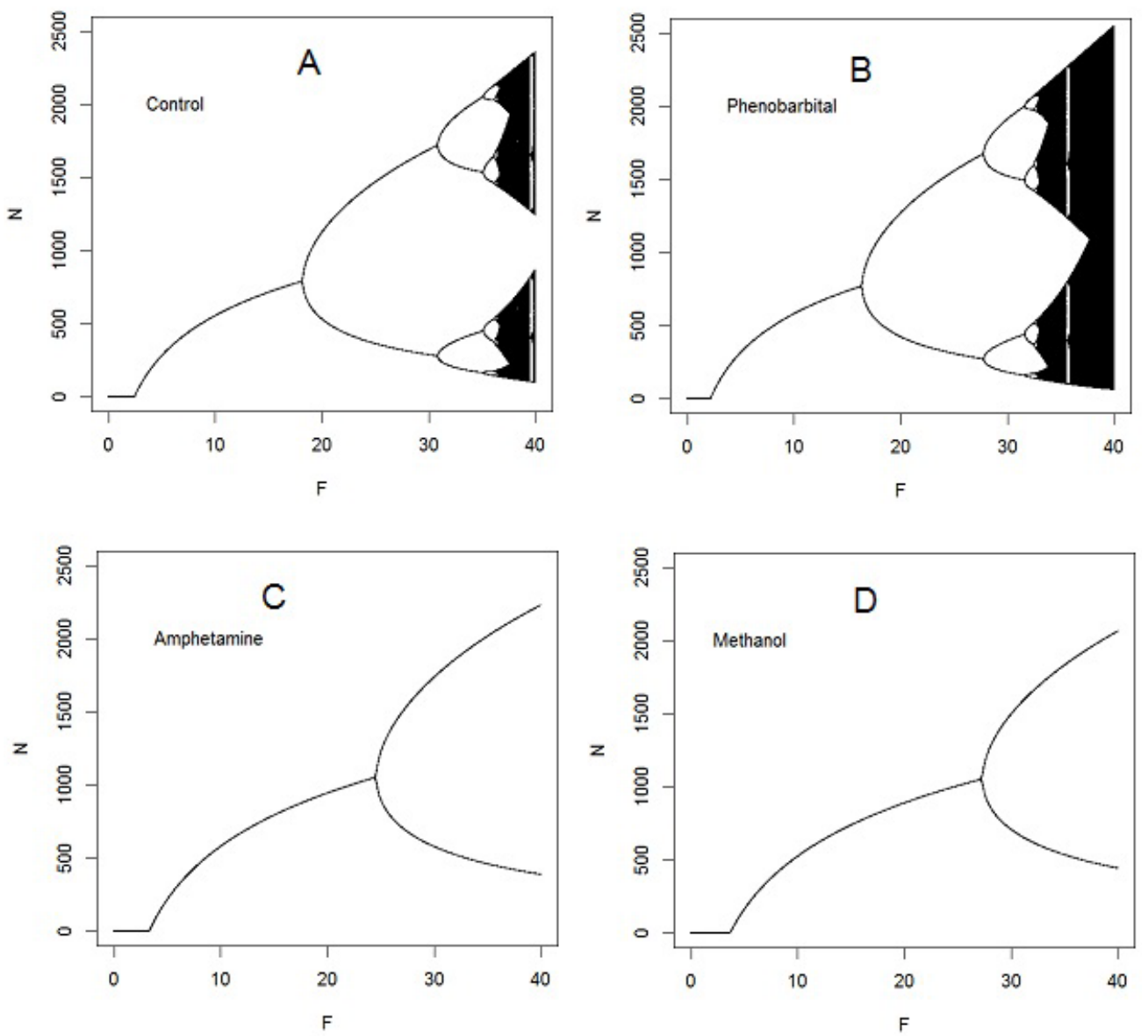

Figure 1: A-D: Bifurcation analysis showing the spectrum of dynamic behaviors produced by the variation in fecundity (F) on the dynamics of Chrysomya albiceps influenced by control (no psychoactive drug), phenobarbital, amphetamine and methanol. 

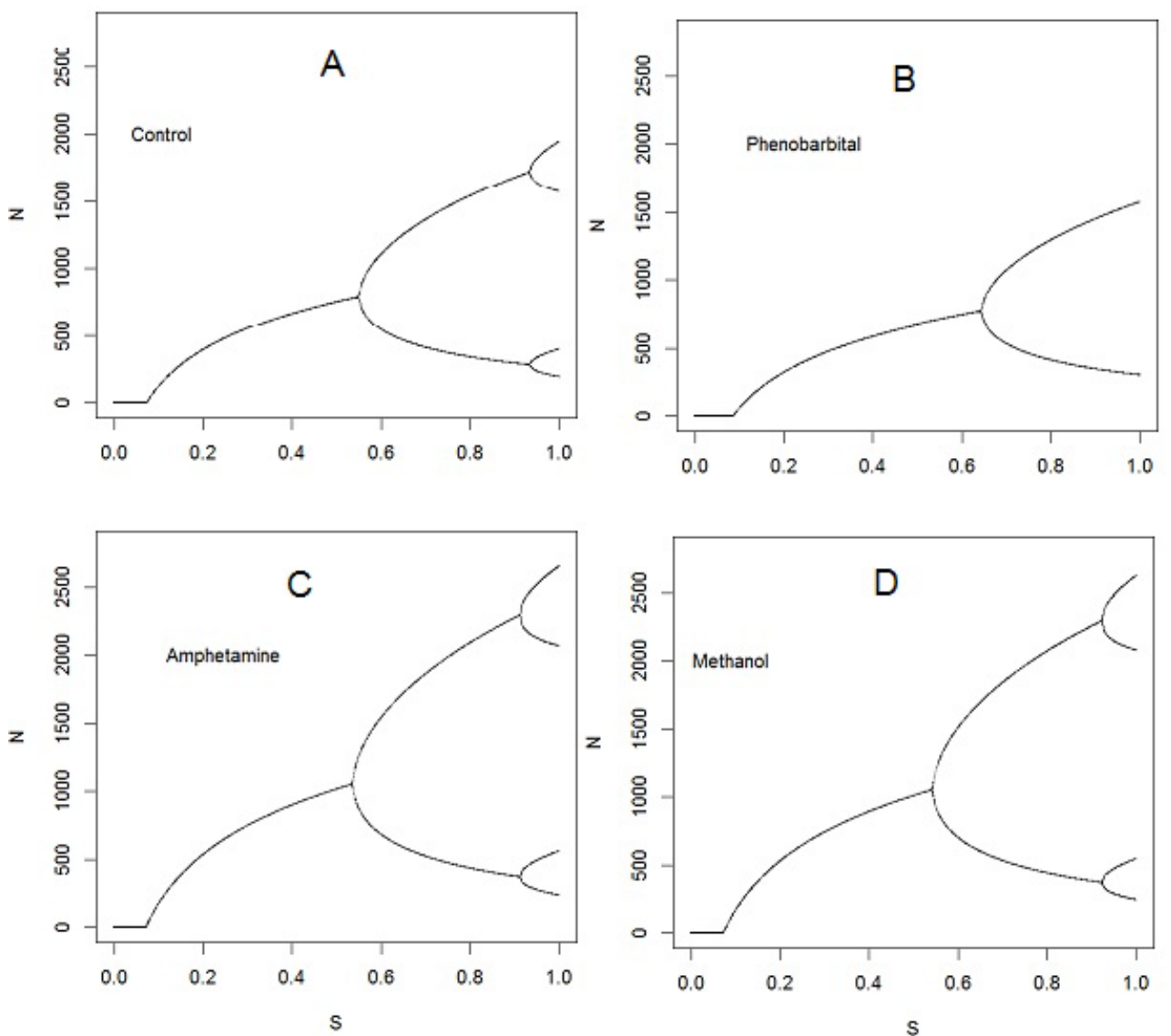

Figure 2: A-D: Bifurcation analysis showing the spectrum of dynamic behaviours produced by the variation in survival (S) on the dynamics of Chrysomya albiceps influenced by control (no psychoactive drug), phenobarbital, amphetamine and methanol.

\begin{tabular}{|c|c|c|c|c|c|c|c|c|}
\hline \multirow{2}{*}{ Variables } & \multicolumn{2}{|c|}{ Control } & \multicolumn{2}{|c|}{ Phenobarbital } & \multicolumn{2}{|c|}{ Methanol } & \multicolumn{2}{|c|}{ Amphetamine } \\
\hline & $\mathrm{F}$ & $S$ & $\mathrm{~F}$ & $S$ & $\mathrm{~F}$ & $S$ & $\mathrm{~F}$ & $\mathrm{~S}$ \\
\hline Y intercepts & 26.74 & 0.81 & 22.87 & 0.90 & 27.12 & 0.54 & 27.45 & 0.60 \\
\hline $\mathrm{RC}$ & 0.0009 & 0.00163 & 0.0006 & 0.002 & 0.0009 & 0.001 & 0.0009 & 0.001 \\
\hline $\mathrm{r}^{2}$ & 0.66 & 0.80 & 0.54 & 0.90 & 0.65 & 0.90 & 0.61 & 0.89 \\
\hline ANOVA & $445^{*}$ & $40.60^{*}$ & $264^{*}$ & $94.64^{*}$ & $414^{*}$ & $80.59^{*}$ & $345^{*}$ & $81.53^{*}$ \\
\hline
\end{tabular}

Table 1: Exponential regression analysis of fecundity and survival for control, phenobarbital, methanol and amphetamine treatments.

Bifurcation diagrams were used to describe the variation in population size due to the parametric space of Chrysomya albiceps fecundity and survival. This analytical tool has been frequently used to investigate the sensitivity of demographic parameters in studies with blow flies [26,39] and with beetles [40]. A bifurcation diagram shows the entire variation of the values of the parameters for a single description [41]. The results obtained for the control experiment indicated that the fecundity bifurcation diagram displayed the entire range of possible behaviours that can be obtained, from stable equilibrium to cycles and up to chaos. The diagram that describes survival as an independent variable shows the same cascading behaviour, but results in, at most, an eight-point limit cycle. Earlier studies with blow flies have found similar results, consistently indicating that fecundity is the demographic parameter with the highest sensitivity for displaying complex behaviours, such as cycles and chaos [28]. Amphetamine and its positive control (methanol) added to the culture medium had a stabilising effect on fecundity compared to the control treatment, since the system was limited to a single bifurcation. Few studies have employed this approach. However, the few existing results indicate changes in population stability. Mullany et al. [42] concluded that methamphetamine accelerates larval growth, increases the size of all life stages, and influences survival and/or fecundity of the blow fly Calliphora stygia (Fabricius).

Amphetamine has also been studied with respect to its capacity to inhibit female insemination, with results providing some evidence of this for the blow fly Phormia regina (Meigen) [43]. Moe et al. [44] demonstrated that sublethal doses of cadmium enhance the stability of population dynamics, which could lead to greater population persistence. However, since the mean densities were reduced in populations treated with cadmium, individuals are more susceptible to extinction through stochastic demographic processes [44]. In the sensitivity analysis of survival in the present study, the treatment with 
amphetamine and its positive control (methanol) gave results similar to that of the control, with up to a four-point limit cycle at the higher values for survival.

The demographic parameters of fecundity and survival are highly dependent on density, and psychoactive or toxic substances may impair the sensitivity of the parameters that govern species dynamics. Forbes et al. [45] reviewed several experiments and found that the experimental design is extremely important for this type of study, and may influence its results. The effects of exposure to toxic substances are too complex to evaluate in the field, because they usually induce various types of interactions between the toxicant and density dependence, mitigating or enhancing the effect on the population. This aspect was decisive in enabling the design and development of this study in the laboratory.

Little is known about the effects of toxicants on density dependence in insects, but some studies, such as that of Gui and Grant [46], have demonstrated the effect of toxicants on density dependence and vice versa. These authors also examined possible compensation mechanisms between the factors as well as the synergistic effects when evaluated jointly. The purpose of the present study was not to evaluate the effect of psychopharmaceuticals on density dependence, but the effect was assessed indirectly through the demographic parameters of fecundity and survival, which are density-dependent functions. As in the study by Gui and Grant [46], we observed the susceptibility of the population to the stress of density dependence, when populations were subjected to the action of the chemical substances. Moe et al. [44] exposed populations of the blow fly Lucilia sericata (Meigen) to cadmium and evaluated survival, among other factors, as was done in the present study. At low densities, the demographic rates declined in response to exposure to cadmium. Even with the high toxicity to Lucilia sericata, when populations were exposed to high densities, the result was positive, contributing to increase the values of the parameters. Thus, it can be stated that there was a density-dependent compensatory reaction. The results of the present study showed that the population dynamics of Chrysomya albiceps larvae is sensitive to the action of psychoactive drugs, with behavioural inversions that can disrupt the configuration of species in a system characterised by necrophagous insects.

Chrysomya tends to breed only on fresh carcasses, and females from a given generation usually breed only once on the same carcass. For this reason, it is commonly assumed that the drugs in carcasses would affect only one generation of blow flies. However, this assumption does not consider that alterations in life-history traits of calliphorid flies caused by psychoactive drugs may be transmitted from parents to offspring through epigenetic transgenerational inheritance. A chemical such as amphetamine in the larval environment may affect the individuals that feed on it, and also their offspring. The $\mathrm{F}_{2}$ and subsequent generations may also be affected, depending on the proximate mechanism that mediates transmission across generations [32]. These mechanisms are still poorly understood, but there is evidence in flies of transmission via changes in chromatin structure [47]. If such an effect does persist for one or more generations, then demographic consequences may result. The long-lasting effects of the possible epigenetic transgenerational inheritance of psychoactive drugs on behaviour and life-history traits in blow flies are poorly known and should be further studied.

\section{Self-Critique}

Number of replications: Because our main interest was to estimate the parameters of the exponential regression, we felt it was more prudent to achieve a better resolution on the $\mathrm{x}$-axis, i.e., to work with more densities than repetitions. This explains the relatively low number of replications used for each experimental condition.

Possible limitation of our results: We only carried out a sensitivity analysis of the parameters as a function of the action of the psychoactive drugs on Chrysomya albiceps. The parameters are sensitive to changes of values, regardless of the cause. One possible cause of variation in the demographic parameters is the action of the drugs.

\section{Acknowledgements}

JZG and TCM were supported by a scholarship from FAPESP (São Paulo Research Foundation), grants 06/59889-3 and 06/60504-9, respectively. WACG was supported by a grant from CNPq (The National Council for Scientific and Technological Development)

\section{Declaration of Conflicting Interests}

The authors declare that they have no conflict of interest.

\section{References}

1. Villet $\mathrm{MH}$, Amendt $\mathrm{J}$ (2011) Advances in entomological methods for death time estimation. In: Turk EE (ed) Forensic Pathology Reviews. Humana Press Heidelberg, 213-237.

2. Thyssen PJ, Souza CM, Shimamoto PM, Salewski TB, Moretti TC (2014) Rates of development of immatures of three species of Chrysomya (Diptera: Calliphoridae) reared in different types of animal tissues: Implications for estimating the postmortem interval. Parasitol Res 113: 3373-3380.

3. Micozzi MS (1986) Experimental study of postmortem change under field conditions: Effects of freezing, thawing, and mechanical injury. J Forensic Sci 31: 953-961.

4. Matuszewski S, Mądra-Bielewicz A (2016) Validation of temperature methods for the estimation of pre-appearance interval in carrion insects. Forensic Sci Med Pathol 12: 50-57.

5. Gosselin M, Wille SMR, Ramirez-Fernandez MM, Di Fazio V, Samyn N, et al. (2011) Entomotoxicology, experimental set-up and interpretation for forensic toxicologists. Forensic Sci Int 208: 1-9.

6. Rezende F, Alonso MA, Souza CM, Thyssen PJ, Linhares AX (2014) Developmental rates of immatures of three Chrysomya species (Diptera Calliphoridae) under the effect of methylphenidate hydrochloride, phenobarbital and methylphenidate hydrochloride associated with phenobarbital. Parasito Res 113: 1897-1907.

7. Smith KE, Wall R (1997) Asymmetric competition between larvae of the blowflies Calliphora vicina and Lucilia sericata in carrion. Ecol Entomol 22: 468474.

8. Tomberlin JK, Mohr R, Benbow ME, Tarone AM, VanLaerhoven S (2011) A roadmap for bridging basic and applied research in forensic entomology. Annu Rev Entomol 56: 401-421.

9. Galindo LA, Moral RA, Moretti TC, Godoy WAC, Demetrio CGB (2016) Intraguild predation influences oviposition behavior of blow flies (Diptera: Calliphoridae) Parasitol Res 115: 2097-2102.

10. Silva EIT, Wilhelmi B, Villet MH (2017) Forensic entomotoxicology revisitedtowards professional standardisation of study designs. Int J Legal Med 131 1399-1412.

11. Bourel B, Hedouin V, Martin-Bouyer L, Becart A, Tournel G, et al. (1999) Effects of morphine in decomposing bodies on the development of Lucilia sericata (Diptera: Calliphoridae). J Forensic Sci 44: 354-358.

12. Carvalho LML, Linhares AX, Trigo JR (2001) Determination of drug levels and the effect of diazepam on the growth of necrophagous flies of forensic importance in southeastern Brazil. Forensic Sci Int 120: 140-144.

13. Gomes G (2006) Self-organized processes: Effects of chemical substances that act on the nervous system on the development and pattern of post-feeding larval dispersal of dipterans (Calliphoridae and Muscidae). MSc Dissertation, São Paulo State University.

14. Goff ML, Omori Al, Goodbrod JR (1989) Effect of cocaine in tissues on the development rate of Boettcherisca peregrina (Diptera: Sarcophagidae). J Med Entomol 26: 91-93.

15. Goff ML, Brown WA, Hewadikaram KA, Omori Al (1991) Effect of heroin in 
Citation: Gião JZ, Reigada C, Moretti TC, Godoy WAC (2017) Effect of Psychoactive Drugs on Demographic Parameters of the Blow Fly Chrysomya albiceps (Wiedemann) (Diptera: Calliphoridae). J Forensic Res 8: 400. doi: 10.4172/2157-7145.1000400

Page 6 of 6

decomposing tissues on the development rate of Boettcherisca peregrina (Diptera, Sarcophagidae) and implications of this effect on estimation of postmortem intervals using arthropod development patterns. J Forensic Sci 36 $537-542$

16. Goff ML, Brown WA, Omori Al, LaPointe DA (1993) Preliminary observations of the effects of amitriptyline in decomposing tissues on the development of Parasarcophaga ruficornis (Diptera: Sarcophagidae) and implications of this effect to estimation of postmortem interval. J Forensic Sci 38: 316-322.

17. Zeferino MT (2004) Traffic accidents and amphetamine-type stimulants: a case study with road haulage companies in the state of Santa Catarina. MSc Dissertation, Federal University of Santa Catarina.

18. Weathermon R, Crabb DW (1999) Alcohol and medication interactions. Alcoho Res Health 23: 40-54.

19. Coupey SM (1997) Barbiturates. Pediatr Rev 18: 260-265.

20. Rios DP, Bastos FM, Cunha LC, Valadares MC (2005) Attempted suicide with the use of drugs registered by the CIT-GO in the years 2003 and 2004. Revista Eletrônica de Farmácia 2: 6-14.

21. Moretti TC, Godoy WAC (2013) Spatio-temporal dynamics and preference for type of bait in necrophagous insects, particularly native and introduced blow flies (Diptera: Calliphoridae). J Med Entomol 50: 415-424.

22. Guimaraes JH, Prado AP, Linhares AX (1978) Three newly introduced blowfly species in Southern Brazil (Diptera: Calliphoridae). Rev Bras Entomol 22: 5360

23. Coutinho RM, Godoy WAC, Kraenkel RA (2012) Integrodifference model for blowfly invasion. Theor Ecol 5: 363-371.

24. Catts EP, Goff ML (1992) Forensic entomology in criminal investigations. Annu Rev Entomol 37: 253-272.

25. Godoy WAC (2007) Dynamics of blowfly populations. Func Ecosyst Commun 1: 129-139.

26. Moretti TC, Coutinho RM, Moral RA, Ferreira CP, Godoy WAC (2013) Quantitative and qualitative dynamics of exotic and native blowflies (Diptera: Calliphoridae) with migrations among municipalities. Community Ecol 14: 249-257.

27. Silva ICR, Mancera PFA, Godoy WAC (2003) Population dynamics of Lucilia eximia (Dipt., Calliphoridae). J Appl Entomol 127: 2-6.

28. Godoy WAC, Zuben FJV, Zuben CJV, Reis SF (2001) Spatio-temporal dynamics and transition from asymptotic equilibrium to bounded oscillations in Chrysomya albiceps (Diptera, Calliphoridae). Mem Inst Oswaldo Cruz 96: 627-634.

29. Rosa GS, Costa MIS, Corrente JE, Silveira LVA, Godoy WAC (2011) Population dynamics, life stage and ecological modeling in Chrysomya albiceps (Wiedemann) (Diptera: Calliphoridae). Neotrop Entomol 40: 181-189.

30. Tabor KL, Fell RD, Brewster CC, Pelzer K, Behonick GS (2005) Effects of antemortem ingestion of ethanol on insect successional patterns and development of Phormia regina (Diptera: Calliphoridae). J Med Entomol 42: 481-489.

31. Chaiwong T, Sukontason KL, Chaisri U, Kurahashi H, Sanford M, et al. (2011)
Effects of human contraceptive on reproduction and offspring in Chrysomya megacephala. Asian Pac J Trop Med 4: 259-265

32. Bonduriansky R, Crean AJ, Day T (2012) The implications of nongenetic inheritance for evolution in changing environments. Evol Appl 5: 192-201.

33. Polkki M, Kangassalo K, Rantala MJ (2012) Transgenerational effects of heavy metal pollution on immune defense of the blow fly Protophormia terraenovae. PLoS One 7: e38832.

34. Leal TTS, Prado AP, Antunes AJ (1982) Rearing the larvae of the blowfly Chrysomya chloropyga (Wiedemann) (Diptera, Calliphoridae) on oligidic diets. Rev Bras Zool 1: 41-44.

35. Estrada DA, Grella MD, Thyssen PJ, Linhares AX (2009) Rate of development of Chrysomya albiceps (Wiedemann) (Diptera: Calliphoridae) in artificial die plus animal tissue for forensic use. Neotrop Entomol 38: 203-207.

36. Linhares AX (1988) The gonotrophic cycle of Chrysomya megacephala (Diptera, Calliphoridae) in the laboratory. Rev Bras Entomol 32: 383-392.

37. Rodriguez DJ (1989) A model of population dynamics for the fruit fly Drosophila melanogaster with density dependence in more than one life stage and delayed density effects. J Anim Ecol 58: 349-365.

38. Prout T, McChesney F (1985) Competition among immatures affects their adult fertility: Population dynamics. Am Nat 126: 521-558.

39. Serra H, Silva ICR, Mancera PFA, Faria LDB, Zuben CJV, et al. (2007) Stochastic dynamics in exotic and native blowflies: an analysis combining laboratory experiments and a two-patch metapopulation model. Ecol Res 22 : 686-695.

40. Cushing JM, Costantino RF, Dennis B, Desharnais RA, Henson SM (2003) Chaos in ecology: Experimental nonlinear dynamics, Theoretical Ecology Series, vol. 1, Academic Press (Elsevier Science), Cambridge.

41. Edelstein-Keshet L (1988) Mathematical models in biology. McGraw-Hill, New York.

42. Mullany C, Keller PA, Nugraha AS, Wallman JF (2014) Effects of methamphetamine and its primary human metabolite, p-hydroxymethamphetamine, on the development of the Australian blowfly Calliphora stygia. Forensic Sci Int 241: 102-111.

43. Evans BP, Stoffolano JG, Yin CM, Meyer JS (1998) The effects of injection of amphetamine on female insemination in the black blow fly, Phormia regina (Diptera: Calliphoridae). Physiol Entomol 23: 20-24.

44. Moe SJ, Stenseth NC, Smith RH (2002) Density-dependent compensation in blowfly populations give indirectly positive effects of a toxicant. Ecology 83 1597-1603.

45. Forbes VE, Sibly RM, Calow $P$ (2001) Toxicant impacts on density-limited populations: A critical review of theory, practice, and results. Ecol Appl 11 1249-1257.

46. Gui Y, Grant A (2008) Joint effects of density dependence and toxicant exposure on Drosophila melanogaster populations. Ecotoxicol Environ Saf 70: 236-243.

47. Ost A, Lempradl A, Casas E, Weigert M, Tiko T, et al. (2014) Paternal diet defines offspring chromatin state and intergenerational obesity. Cell 159: 1352 1364. 